\title{
Pathologic separation of idiopathic pulmonary fibrosis from fibrotic hypersensitivity pneumonitis
}

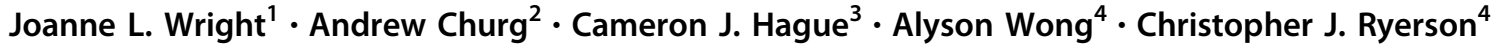

Received: 1 July 2019 / Revised: 22 September 2019 / Accepted: 24 September 2019 / Published online: 28 October 2019

(c) The Author(s), under exclusive licence to United States \& Canadian Academy of Pathology 2019

\begin{abstract}
Accurate separation of idiopathic pulmonary fibrosis from fibrotic (chronic) hypersensitivity pneumonitis is crucial to patient management, but is frequently a difficult problem. Our objective was to identify pathologic variables that help make this separation. Clinical, radiological, and pathologic data were re-reviewed for 23 patients with a fibrotic interstitial lung disease and biopsy suggesting idiopathic pulmonary fibrosis or fibrotic hypersensitivity pneumonitis. Clinical features, high-resolution computed tomography, and surgical lung biopsies were each examined independently using a prespecified approach. This was followed by a multidisciplinary discussion in which the likelihood of an idiopathic pulmonary fibrosis diagnosis was assigned by the clinician alone based only on clinical data, by the clinician and radiologist based on integrated clinical and radiologic data, and by the clinician, radiologist, and pathologist based on all three domains. A higher multidisciplinary discussion-based confidence of idiopathic pulmonary fibrosis was associated with older age at diagnosis, male sex, higher forced vital capacity, and absence of ground glass changes. Pathologic variables associated with a higher multidisciplinary discussion-based confidence of idiopathic pulmonary fibrosis included increased number of fibroblast foci $/ \mathrm{cm}^{2}$ and increased subpleural fibrosis. Pathologic variables associated with a higher multidisciplinary discussion-based confidence of hypersensitivity pneumonitis included an increased fraction of bronchioles with peribronchiolar metaplasia, increased foci of peribronchiolar metaplasia $/ \mathrm{cm}^{2}$, and presence of giant cells/granulomas. These results provide guidance in separating idiopathic pulmonary fibrosis from hypersensitivity pneumonitis; however, a third of cases could not be confidently classified even when using these pathologic features combined with clinical and radiologic information in a multidisciplinary discussion.
\end{abstract}

\section{Introduction}

Accurate diagnosis of fibrotic interstitial lung disease requires multidisciplinary integration of clinical, radiological, and pathologic information [1]; however, diagnostic uncertainty

$\triangle$ Andrew Churg

achurg@mail.ubc.ca

1 Department of Pathology, University of British Columbia \& St Paul's Hospital, Vancouver, BC, Canada

2 Department of Pathology, University of British Columbia \& Vancouver General Hospital, Vancouver, BC, Canada

3 Department of Radiology, St Paul's Hospital, Vancouver, BC, Canada

4 Department of Medicine, University of British Columbia \& Centre for Heart Lung Innovation, St. Paul's Hospital, Vancouver, BC, Canada frequently remains following a thorough evaluation [2]. The existence of specific diagnostic criteria for some interstitial lung disease subtypes reduces, but does not eliminate, the number of patients who are unable to be provided a confident diagnosis [3].

One area of particular difficulty is distinguishing between idiopathic pulmonary fibrosis and fibrotic (chronic) hypersensitivity pneumonitis [4], and this separation is critical given their different treatment approaches and prognosis. The poor reported interobserver agreement across experienced multidisciplinary teams for the diagnosis of hypersensitivity pneumonitis suggests a lack of agreement on the specific features that define hypersensitivity pneumonitis and those that distinguish it from the pathological pattern of usual interstitial pneumonia that is characteristic of idiopathic pulmonary fibrosis (i.e., a pathologic pattern of "usual interstitial pneumonia/idiopathic pulmonary fibrosis") [5-7]. Despite identification of some pathologic findings that may aid this separation of idiopathic pulmonary fibrosis [8-10], 
to our knowledge these features have never been tested in a formal multidisciplinary discussion setting. In this study we have used a multidisciplinary discussion and diagnostic likelihood approach to identify pathological variables that distinguish the clinical diagnoses of idiopathic pulmonary fibrosis and chronic hypersensitivity pneumonitis.

\section{Methods}

\section{Study overview}

This retrospective cross-sectional study was approved by the Research Ethics Board of the University of British Columbia (H17-01740). Patients with a surgical lung biopsy-supported diagnosis of idiopathic pulmonary fibrosis or hypersensitivity pneumonitis were identified from a prospective single-center database and then re-reviewed without knowledge of the previous diagnosis, therapeutic choices, or disease behavior that occurred after the biopsy. Clinical, radiological, and pathologic data were respectively reviewed in isolation by a pulmonologist, a chest radiologist, and two pathologists, all of whom have expertise in interstitial lung disease and $>7$ years of clinical experience participating in a multidisciplinary intersitial lung disease conference. Before starting the review, we identified a core set of clinical, radiological, and pathologic variables to be recorded, emphasizing variables that were believed to be of value in diagnosing idiopathic pulmonary fibrosis or hypersensitivity pneumonitis based on clinical experience and review of previous literature [3, 11, 12]. The diagnostic likelihood of hypersensitivity pneumonitis or idiopathic pulmonary fibrosis was assigned by the clinician alone based only on clinical data and then by clinician and radiologist based on integrated review and discussion of clinical and radiological data. The final multidisciplinary discussion-based diagnostic confidence was then assigned by consensus based on all three domains, and variables associated with the diagnostic confidence of idiopathic pulmonary fibrosis and hypersensitivity pneumonitis were then identified. The study design is summarized in Fig. 1.

\section{Study population}

All included patients had evidence of fibrosis on highresolution computed tomography and a surgical lung biopsy that was previously reported as suggestive of either idiopathic pulmonary fibrosis or fibrotic hypersensitivity pneumonitis (hereafter referred to as hypersensitivity pneumonitis). Patients with a connective tissue disease were excluded, as were patients meeting proposed criteria for interstitial pneumonia with autoimmune features [13].

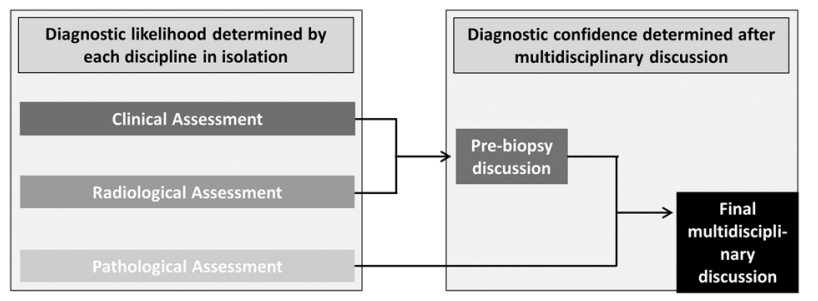

Fig. 1 Overview of study design

\section{Clinical data}

Predefined clinical variables were extracted from the patient chart by an interstitial lung disease clinician who did not participate in the multidisciplinary discussion (AW), including baseline data and longitudinal features that predated the performance of the surgical lung biopsy. These included age at the time of biopsy, sex, race, smoking history (current/previous/never and number of pack-years), potential exposures, auscultatory crackles or wheeze, forced vital capacity, diffusion capacity of the lung for carbon monoxide, and potential exposures. Clinical data that were obtained following performance of the lung biopsy, for example, patient outcome, were not made available for multidisciplinary discussion.

\section{Radiological data}

Predefined radiological variables were assessed based on a re-review of imaging performed prior to the performance of a surgical lung biopsy. These included distribution (lower lobe predominance, subpleural, and/or peribronchovascular) as well as the presence or absence of honeycombing (above and/or below the carina), pure ground glass, mosaic attenuation with lobular sharply defined air trapping on expiratory computed tomography, nodules, consolidation (not present in any patient), and cysts unrelated to honeycombing. All radiologic features were recorded as binary variables

\section{Pathologic data}

Predefined pathologic variables were assessed based on a re-review of two representative slides from each biopsied lobe. All biopsies were fixed by formalin inflation. The area of the tissue on each slide was measured using image analysis for variables that could be quantified in this manner. For dichotomous variables the presence of a feature in any lobe was considered to be present for that patient even if absent from other lobes; the data from all lobes were averaged for continuous variables. The following variables were examined: fraction of bronchioles with peribronchiolar metaplasia (peribronchiolar metaplasia) and number of foci 
of peribronchiolar metaplasia per $\mathrm{cm}^{2}$, number of fibroblast foci per $\mathrm{cm}^{2}$ and their location (peribronchiolar vs. subpleural/paraseptal), fraction of bronchioles with fibroblast foci, presence of giant cells or granulomas (whether interstitial or airspace), fraction of isolated bronchioles with peribronchiolar (centrilobular) fibrosis (i.e., membranous or respiratory bronchioles with peribronchiolar fibrosis that did not connect to the subpleural region or to an interlobular septum), number of lymphoid aggregates per $\mathrm{cm}^{2}$ and the number with germinal centers, nature and intensity of the interstitial inflammatory infiltrate, fibrosis that bridged from bronchioles to the pleura or interlobular septa, and the presence of areas of normal pleura with nearby bronchioles showing peribronchiolar (centrilobular) fibrosis.

\section{Morphometric measurements}

In addition, each membranous and respiratory bronchiole was identified and analyzed using standardized morphometric principles [14]. For each airway, a point was identified on the wall, and using the role of two dice, the o'clock position of this point was determined. Using this point as a reference, the thickness of the airway wall was measured at four sites around the circumference (12, 3, 6, and 9 o'clock), with a mean and coefficient of variation calculated for each case. These numbers provided a quantitative measure of peribronchiolar fibrosis, as opposed to the simple qualitative assessment of whether bronchioles had peribronchiolar fibrosis described above. The amount and variability of subpleural fibrosis was quantified using the mean and coefficient of variation, with measurements taken at $1 \mathrm{~mm}$ distances along the entire pleural surface.

\section{Assignment of diagnostic likelihood}

The clinician, radiologist, and pathologists participating in the multidisciplinary discussion were blinded to all data that were acquired following the biopsy, including the initial diagnosis, therapeutic choices, and disease behavior. Each participant in the multidisciplinary discussion first assigned their isolated percent-likelihood of a clinical idiopathic pulmonary fibrosis vs. hypersensitivity pneumonitis diagnosis based on data specific to his or her specialty, without input from the other domains. Following this, the clinician and radiologist assigned a consensus prebiopsy percentconfidence of an idiopathic pulmonary fibrosis vs. hypersensitivity pneumonitis diagnosis based on integration of clinical and radiological data, supported by discussion of relevant details from these domains. The clinician, radiologist, and pathologists then conducted a full multidisciplinary discussion and again assigned a consensus post-biopsy percent-confidence of an idiopathic pulmonary fibrosis vs. hypersensitivity pneumonitis diagnosis based on integration of all clinical, radiological, and pathological data that were available at the time of biopsy. For each step, the assigned percent of idiopathic pulmonary fibrosis and hypersensitivity pneumonitis was required to sum to $100 \%$, forcing the assumption that alternative differential diagnoses had been excluded. For each step, the assignment of the percent was based on a gestalt impression after considering all relevant data, similar to the concept applied in a recent survey evaluating diagnostic accuracy of a clinical diagnosis of idiopathic pulmonary fibrosis [15].

\section{Statistical analysis}

Data are described as number (\%) or median (range). Findings are reported according to strata of final multidisciplinary discussion-based diagnostic confidence as previously reported [16], subcategorizing as a high-confidence diagnosis of idiopathic pulmonary fibrosis ( $\geq 70 \%$ confidence), high-confidence diagnosis of hypersensitivity pneumonitis ( $\geq 70 \%$ confidence), and patients with a lowconfidence diagnosis ( $<70 \%$ confidence in either diagnosis). The association of clinical, radiological, and pathological findings with diagnostic likelihood/confidence was determined based on a Spearman correlation or Wilcoxon rank sum test. Multivariable analyses were not performed given the small sample size. All data analysis was performed using Stata version 15.1 (College Station, TX, USA).

\section{Results}

\section{Patient characteristics}

The study population included 23 patients. Baseline clinical and radiologic characteristics of each patient are summarized in Table 1. The number of biopsies per case varied from 1 to 3 (median 2), and were mostly taken from upper and lower lobes. The final multidisciplinary discussionbased diagnostic confidence of idiopathic pulmonary fibrosis ranged from 0 to $95 \%$, with diagnostic confidence of hypersensitivity pneumonitis conversely ranging from 5 to $100 \%$. Table 1 also documents limited data on physiologic changes post biopsy and outcome. These data are included for interest, but were not available to the multidisciplinary discussion participants and were not used in formulating the diagnostic likelihood.

\section{Association of clinical and radiological findings with prebiopsy diagnostic likelihood}

Based only on clinical data, a higher likelihood of an idiopathic pulmonary fibrosis diagnosis was associated with older age at diagnosis, male sex, and higher FVC-\% 
Table 1 Baseline clinical, radiological, and pathological characteristics

\begin{tabular}{|c|c|c|c|c|}
\hline Characteristic & $\begin{array}{l}\text { Full cohort } \\
(N=23)\end{array}$ & $\begin{array}{l}\text { Confident idiopathic } \\
\text { pulmonary fibrosis } \\
(N=5)\end{array}$ & $\begin{array}{l}\text { Low confidence } \\
(N=8)\end{array}$ & $\begin{array}{l}\text { Confident } \\
\text { hypersensitivity } \\
\text { pneumonitis } \\
(N=10)\end{array}$ \\
\hline \multicolumn{5}{|l|}{ Clinical features } \\
\hline Age, year & $63(57-65)$ & $63(63-65)$ & $65(61-68)$ & $58(53-62)$ \\
\hline Male sex & $13(57 \%)$ & $5(100 \%)$ & $5(63 \%)$ & $3(30 \%)$ \\
\hline \multicolumn{5}{|l|}{ Smoking history } \\
\hline Never-smoker & $10(43 \%)$ & $1(20 \%)$ & $2(25 \%)$ & $7(70 \%)$ \\
\hline Past or current smoker & $13(57 \%)$ & $4(80 \%)$ & $6(75 \%)$ & $3(30 \%)$ \\
\hline Pack-years & $2(0-25)$ & $20(13-20)$ & $2.5(1-19)$ & $0(0-23)$ \\
\hline Potential exposure & $15(65 \%)$ & $2(40 \%)$ & $4(25 \%)$ & $9(50 \%)$ \\
\hline Avian antigen ${ }^{a}$ & 9 & 2 & 4 & 3 \\
\hline Mold & 6 & 1 & 0 & 5 \\
\hline Other $^{\mathrm{b}}$ & 2 & 0 & 0 & 4 \\
\hline \multicolumn{5}{|l|}{ Physiologic features } \\
\hline Forced vital capacity $\%$-predicted & $77(66-86)$ & $93(92-95)$ & $77(66-80)$ & $73(62-82)$ \\
\hline Diffusing capacity $\%$-predicted & $52(50-61)$ & $59(55-68)$ & $50(44-52)$ & $55(51-64)$ \\
\hline \multicolumn{5}{|l|}{ Radiologic features } \\
\hline Lower lobe predominant & $19(83 \%)$ & $5(100 \%)$ & $6(75 \%)$ & $8(80 \%)$ \\
\hline Honeycombing below carina & $7(30 \%)$ & $3(60 \%)$ & $3(38 \%)$ & $1(10 \%)$ \\
\hline Ground glass opacities & $14(61 \%)$ & $1(20 \%)$ & $6(75 \%)$ & $7(70 \%)$ \\
\hline \multicolumn{5}{|l|}{ Pathologic features } \\
\hline $\begin{array}{l}\text { Fraction of bronchioles with peribronchiolar } \\
\text { metaplasia }\end{array}$ & $0.16(0.09-0.57)$ & $0.15(0-0.17)$ & $0.15(0-0.38)$ & $0.40(0.13-0.72)$ \\
\hline Foci of peribronchiolar metaplasia per $\mathrm{cm}^{2}$ & $0.76(0.24-1.48)$ & $0.31(0-0.35)$ & $0.68(0-1.48)$ & $1.32(0.28-2.16)$ \\
\hline Subpleural fibrosis morphologic mean & $36(28-42)$ & $36(35-36)$ & $37(31-43)$ & $28(21-41)$ \\
\hline Presence of bridging fibrosis & $15(65 \%)$ & $3(60 \%)$ & $6(75 \%)$ & $6(60 \%)$ \\
\hline Number of fibroblastic foci per $\mathrm{cm}^{2}$ & $2.1(1.1-4.4)$ & $3.3(2.4-6.0)$ & $3.4(1.8-5.1)$ & $1.1(0.4-1.6)$ \\
\hline Giant cells or granulomas & $10(43 \%)$ & $0(0 \%)$ & $2(25 \%)$ & $8(80 \%)$ \\
\hline \multicolumn{5}{|l|}{ Patient outcomes ${ }^{c}$} \\
\hline Forced vital capacity decline $\geq 10 \%$ per year $^{\mathrm{d}}$ & $5(22 \%)$ & $0(0 \%)$ & $3(38 \%)$ & $2(20 \%)$ \\
\hline Lung transplantation & $3(13 \%)$ & $0(0 \%)$ & $2(25 \%)$ & $1(10 \%)$ \\
\hline Deceased & $9(39 \%)$ & $3(60 \%)$ & $3(38 \%)$ & $3(30 \%)$ \\
\hline Median time to death or transplant (years) ${ }^{4}$ & 3.72 & 3.00 & 3.15 & 4.98 \\
\hline
\end{tabular}

Data shown are median (interquartile range) or number (percent) for the full cohort and stratified by the final multidisciplinary discussion diagnostic confidence

The patient outcome data were not available to the multidisciplinary discussion participants

a Patients could report exposure to multiple individual antigens

${ }^{\mathrm{b}}$ Other exposures included standing water, soil, and cement

${ }^{\mathrm{c}}$ At time of most recent follow-up

${ }^{\mathrm{d}}$ During the 2 years following surgical lung biopsy

predicted. Based only on isolated imaging findings, a higher likelihood of an idiopathic pulmonary fibrosis diagnosis was associated with lower lobe-predominant abnormalities, honeycombing below the carina, and the absence of ground glass opacities. The associations of baseline clinical and radiological characteristics with the combined clinicalradiological prebiopsy likelihood are shown in Table 2.

\section{Association of individual pathological findings with blinded biopsy-based diagnostic likelihood}

The associations of individual pathologic variables with blinded biopsy-based diagnostic likelihood are shown in Table 3. Based only on pathologic findings, a higher likelihood of a pathologist-assigned likelihood of an idiopathic 
Table 2 Association of baseline characteristics with prebiopsy diagnostic likelihood of idiopathic pulmonary fibrosis

\begin{tabular}{llll}
\hline Domain & Variable & $r$ value or median difference & $p$ value \\
\hline Clinical features & Age at diagnosis & 0.47 & 0.02 \\
& Male sex & $35(10$ to 55$)$ & 0.01 \\
& Smoking pack-years & 0.23 & 0.29 \\
& Potential exposure & $0(-30$ to 20$)$ & 0.85 \\
& Forced vital capacity, \%-predicted & 0.50 & 0.02 \\
& Diffusing capacity, \%-predicted & 0.25 & 0.24 \\
Radiological features & Lower lobe predominant & $31(10$ to 65$)$ & 0.02 \\
& Subpleural & $33(-10$ to 80$)$ & 0.15 \\
& Honeycombing above carina & $20(-15$ to 50$)$ & 0.29 \\
& Honeycombing below carina & $35(0$ to 60$)$ & 0.03 \\
& Ground glass & $-30(-55$ to -5$)$ & 0.02 \\
& Nodules & $-8(-35$ to 20$)$ & 0.54 \\
& Mosaic attenuation & $-15(-45$ to 15$)$ & 0.33 \\
& Peribronchovascular & $-10(-40$ to 15$)$ & 0.42 \\
& Cysts & $0(-65$ to 65$)$ & 1.00 \\
& Consolidation & n/a & n/a \\
\hline
\end{tabular}

For continuous variables, data shown are $r$ and $p$ values obtained by Spearman correlation. For dichotomous variables, data shown are median difference (range) in the percent likelihood of an idiopathic pulmonary fibrosis diagnosis for patients with the feature compared to patients without the feature, with corresponding $p$ value from a Wilcoxon rank sum test. Variables positively associated with a higher diagnostic likelihood of idiopathic pulmonary fibrosis are shown as positive $r$ values or a positive median difference

Table 3 Association of pathologic variables with the likelihood of idiopathic pulmonary fibrosis

\begin{tabular}{|c|c|c|c|c|}
\hline \multirow[t]{2}{*}{ Variable } & \multicolumn{2}{|c|}{$\begin{array}{l}\text { Blinded biopsy-based likelihood of } \\
\text { idiopathic pulmonary fibrosis }\end{array}$} & \multicolumn{2}{|c|}{$\begin{array}{l}\text { Final multidisciplinary discussion- } \\
\text { based confidence of idiopathic } \\
\text { pulmonary fibrosis }\end{array}$} \\
\hline & $r$ value or median difference & $p$ value & $r$ value or median difference & $p$ value \\
\hline Fraction of bronchioles with peribronchiolar metaplasia & -0.38 & 0.08 & -0.51 & 0.02 \\
\hline Foci of peribronchiolar metaplasia $/ \mathrm{cm}^{2}$ & -0.46 & 0.02 & -0.55 & 0.01 \\
\hline Number of fibroblast foci $/ \mathrm{cm}^{2}$ & 0.60 & 0.002 & 0.68 & 0.0003 \\
\hline $\begin{array}{l}\text { Paraseptal/subpleural fibroblast foci vs. peribronchiolar } \\
\text { (centrilobular) }\end{array}$ & $0(-22$ to 39$)$ & 1.00 & $-15(-40$ to 10$)$ & 0.18 \\
\hline Fraction of bronchioles with fibroblast foci & -0.35 & 0.10 & 0.03 & 0.88 \\
\hline Giant cells or granulomas & $-39(-65$ to -16$)$ & 0.004 & $-30(-50$ to -10$)$ & 0.003 \\
\hline Fraction of isolated bronchioles with peribronchiolar fibrosis & -0.58 & 0.005 & -0.37 & 0.09 \\
\hline Radial estimate of fibrosis for respiratory bronchioles & 0.29 & 0.20 & -0.06 & 0.81 \\
\hline Radial estimate of fibrosis for membranous bronchioles & 0.47 & 0.03 & 0.33 & 0.15 \\
\hline Subpleural fibrosis morphometric mean & 0.42 & 0.06 & 0.49 & 0.02 \\
\hline Bridging fibrosis & $-17(-56$ to 7$)$ & 0.12 & $0(-20$ to 25$)$ & 0.90 \\
\hline $\begin{array}{l}\text { Normal pleura juxtaposed to peribronchiolar (centrilobular) } \\
\text { fibrosis }\end{array}$ & $-41(-64$ to 14$)$ & 0.006 & $-25(-40$ to 0$)$ & 0.06 \\
\hline
\end{tabular}

The blinded biopsy-based likelihood of idiopathic pulmonary fibrosis refers to the likelihood of IPF based on isolated inspection of the surgical lung biopsy specimen, without consideration of any clinical or radiological findings. The final multidisciplinary discussion-based confidence of idiopathic pulmonary fibrosis refers to the confidence of an idiopathic pulmonary fibrosis diagnosis based on a complete multidisciplinary discussion that considered all available clinical, radiological, and pathological findings. For continuous variables, data shown are $r$ and $p$ values obtained by Spearman correlation. For dichotomous variables, data shown are median difference (range) in the percent likelihood/confidence of an idiopathic pulmonary fibrosis diagnosis for patients with the feature compared to patients without the feature, with corresponding $p$ value from a Wilcoxon rank sum test. Variables positively associated with a higher diagnostic likelihood of idiopathic pulmonary fibrosis are shown as positive $r$ values or a positive median difference; variables associated with a diagnosis of hypersensitivity pneumonitis are shown as negative numbers 
Fig. 2 Features associated with likelihood/confidence of an idiopathic pulmonary fibrosis diagnosis at each step of the multidisciplinary discussion review

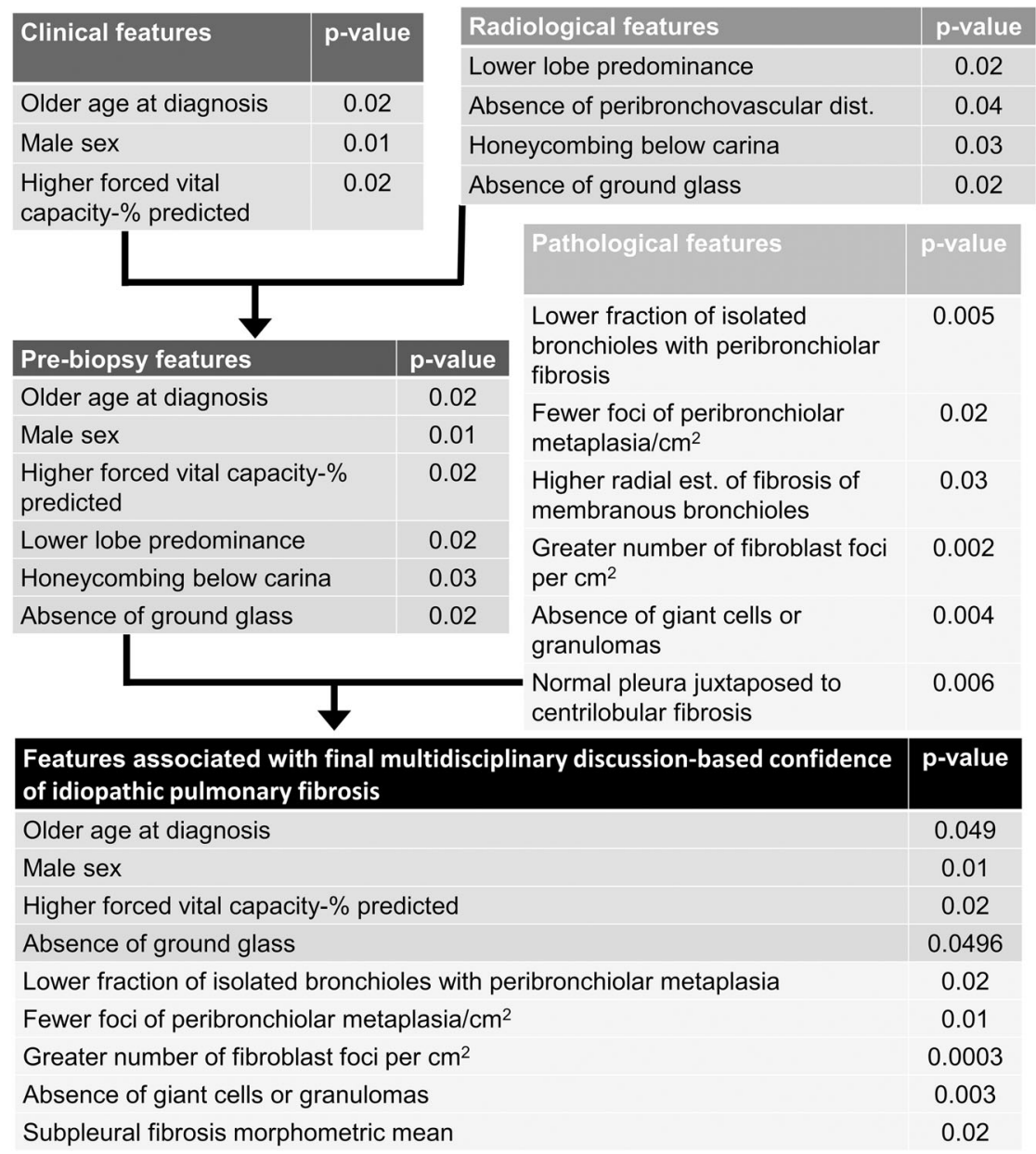

pulmonary fibrosis diagnosis was associated with an increased number of fibroblast foci and increased fibrosis around membranous bronchioles. An increase in the amount of subpleural fibrosis also favored an idiopathic pulmonary fibrosis diagnosis, but was not statistically significant. A higher pathologist-assigned likelihood of hypersensitivity pneumonitis was associated with increased number of foci of peribronchiolar metaplasia $/ \mathrm{cm}^{2}$, presence of giant cells or granulomas, fraction of isolated bronchioles with peribronchiolar fibrosis, and having normal pleura juxtaposed to centrilobular fibrosis. The fraction of bronchioles with peribronchiolar metaplasia also favored a diagnosis of hypersensitivity pneumonitis, but was not statistically significant.

\section{Association of clinical, radiological, and pathologic findings with final multidisciplinary discussion- based diagnostic confidence}

The associations of clinical, radiological, and pathological findings with the final multidisciplinary discussion-based diagnostic confidence are shown in Figs. 2 and 3 and Table 3. A higher multidisciplinary discussion-based confidence of idiopathic pulmonary fibrosis was associated with older age at diagnosis, male sex, higher forced vital capacity \%-predicted, and absence of ground glass changes. Pathologic variables associated with a higher multidisciplinary discussion-based confidence of idiopathic pulmonary fibrosis included increased number of fibroblast foci $/ \mathrm{cm}^{2}$ and increased subpleural fibrosis (Fig. 4). Pathological variables associated with a higher multidisciplinary discussion-based confidence of hypersensitivity pneumonitis included an increased fraction of bronchioles with peribronchiolar metaplasia, increased number of foci of peribronchiolar metaplasia $/ \mathrm{cm}^{2}$, and the presence of giant cells or granulomas (Fig. 4).

The amount of morphometrically measured fibrosis around membranous or respiratory bronchioles (Fig. 4), the coefficient of variation of these measures, the fraction of bronchioles with fibroblast foci (Fig. 4), the fraction of bronchioles with peribronchiolar fibrosis, the presence of bridging fibrosis, and the number of lymphoid aggregates were not associated with either diagnosis. The presence of normal pleura juxtaposed to bronchioles with peribronchiolar fibrosis (Fig. 4) was more common in hypersensitivity pneumonitis but the difference from idiopathic pulmonary fibrosis did not quite achieve significance $(p=0.06)$. Interstitial cellularity was minimal to mild (Fig. 4) in all cases and no case had a 


\section{Clinical Features}

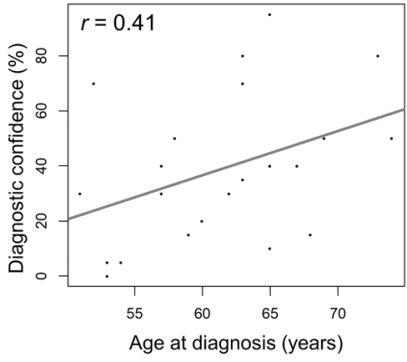

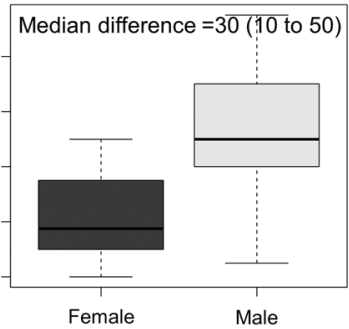

Male

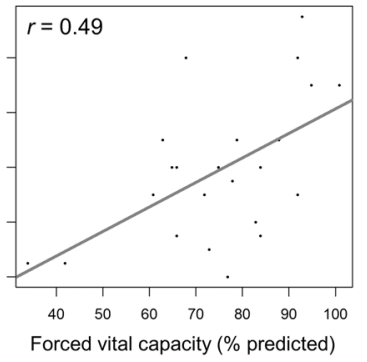

Radiological Features

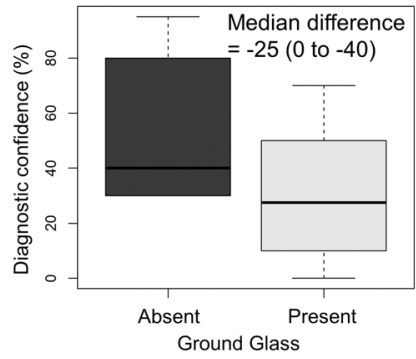

\section{Pathological Features}
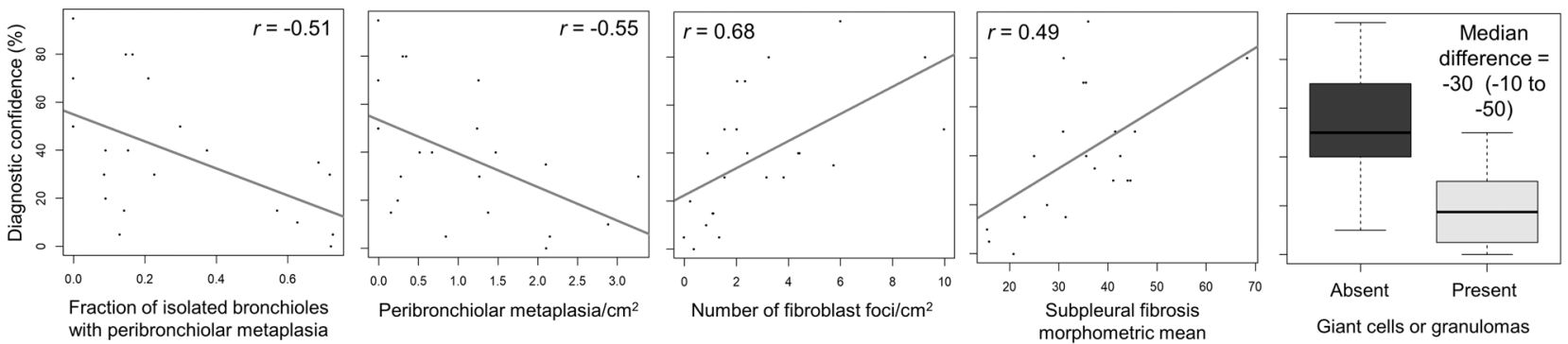

Fig. 3 Unadjusted association of clinical, radiological, and pathological features with final multidisciplinary discussion-based confidence of idiopathic pulmonary fibrosis

predominance of plasma cells. No case had germinal centers. The absence of these latter features provides reassurance that cases of connective tissue disease-associated interstitial lung disease had been excluded from the study.

\section{Discussion}

The separation of idiopathic pulmonary fibrosis from hypersensitivity pneumonitis is frequently challenging. While there is reasonably good agreement about the features of idiopathic pulmonary fibrosis [3,7], the clinical, radiological, and pathologic features that support a diagnosis of hypersensitivity pneumonitis are less established $[6,17,18]$. Clinically and radiologically evident cases of idiopathic pulmonary fibrosis or hypersensitivity pneumonitis are typically not biopsied [3, 7]; however, surgical lung biopsies are frequently performed in more challenging patients with diagnostic uncertainty. The pathologic features in these two conditions can be very similar and there is disagreement in the pathology literature about which features favor each diagnosis $[8,10,19]$. These challenges are illustrated by a recent study of seven experienced multidisciplinary discussion groups who reviewed 70 interstitial lung disease cases and in aggregate showed a high weighted kappa across groups of 0.71 for a diagnosis of idiopathic pulmonary fibrosis, but only 0.29 for hypersensitivity pneumonitis [6], suggesting that there is currently very little agreement in the approach to diagnosing hypersensitivity pneumonitis, even among experts. The distinction between idiopathic pulmonary fibrosis and hypersensitivity pneumonitis has become more crucial given the diverging treatment approaches of these two conditions, with idiopathic pulmonary fibrosis treated with antifibrotic therapies (pirfenidone, nintedanib) and avoidance of immunosuppression (steroids, mycophenolate, azathioprine), while only immunosuppressive therapy is used in hypersensitivity pneumonitis.

In this study, we performed a retrospective multidisciplinary assessment of patients with a diagnosis of idiopathic pulmonary fibrosis or hypersensitivity pneumonitis who had undergone a surgical lung biopsy, with the intent of clarifying the clinical, radiological, and pathologic features that allow better separation of these two entities. We adapted established methods for assigning diagnostic likelihood/confidence at each step of the multidisciplinary discussion process $[15,16,20]$, first based on each domain in isolation, and then based on a full multidisciplinary discussion. This approach acknowledges that the diagnosis of a particular interstitial lung disease subtype is often not dichotomous, but rather falls on a spectrum of diagnostic certainty. This differs considerably from the usual method of pathologic investigation, in which entities that are clearly "A" are compared to entities that are clearly "B". That approach works well for tumors, which tend to be clearly defined, but less well for interstitial lung disease, where securing a specific confident diagnosis is more problematic. The importance of this approach to the diagnosis of 


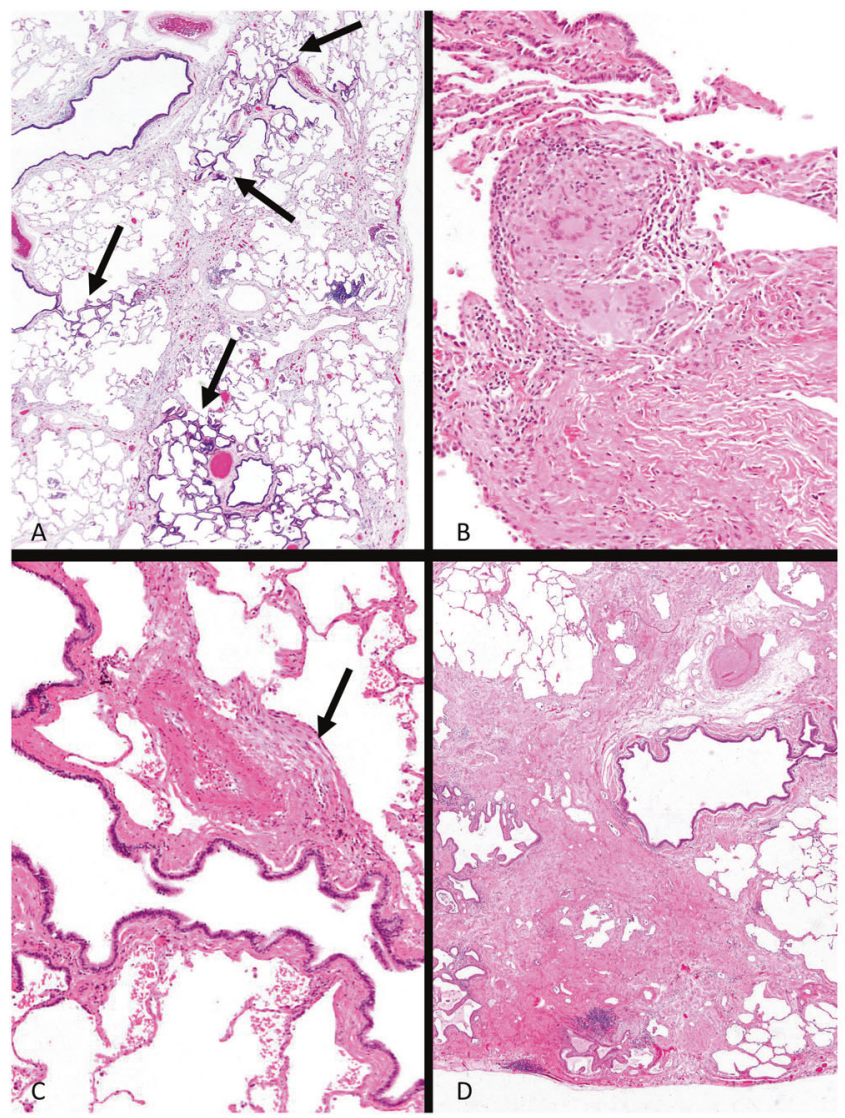

Fig. 4 Examples of pathologic findings. a Extensive peribronchiolar metaplasia (arrows) in hypersensitivity pneumonitis. Peribronchiolar metaplasia affecting more than half of the bronchioles strongly favored a diagnosis of hypersensitivity pneumonitis, but the finding of occasional foci of peribronchiolar metaplasia was not helpful. b An interstitial granuloma in hypersensitivity pneumonitis. Giant cells/ granulomas were only seen in cases with a likelihood of $50 \%$ or greater favoring hypersensitivity pneumonitis in this study. c A fibroblast focus, here next to a bronchiole. Idiopathic pulmonary fibrosis cases had statistically greater numbers of fibroblast foci, but there were no differences in the location of fibroblast foci between hypersensitivity pneumonitis and idiopathic pulmonary fibrosis cases. d Extensive subpleural fibrosis in idiopathic pulmonary fibrosis.

interstitial lung disease was recently endorsed by an international working group [16]; however, previous studies have not evaluated individual pathologic features or overall biopsy impression in this fashion. Using this approach, we identified several clinical, radiological, and pathologic findings that are associated with the final diagnostic confidence of idiopathic pulmonary fibrosis or hypersensitivity pneumonitis, thus specifying key features that should be considered in clinical practice and for incorporation in future diagnostic criteria.

We found that the presence of giant cells or granulomas and extensive peribronchiolar metaplasia supported a diagnosis of hypersensitivity pneumonitis. These two variables are particularly useful to the pathologist because they are easy to observe and quantify. Giant cells and

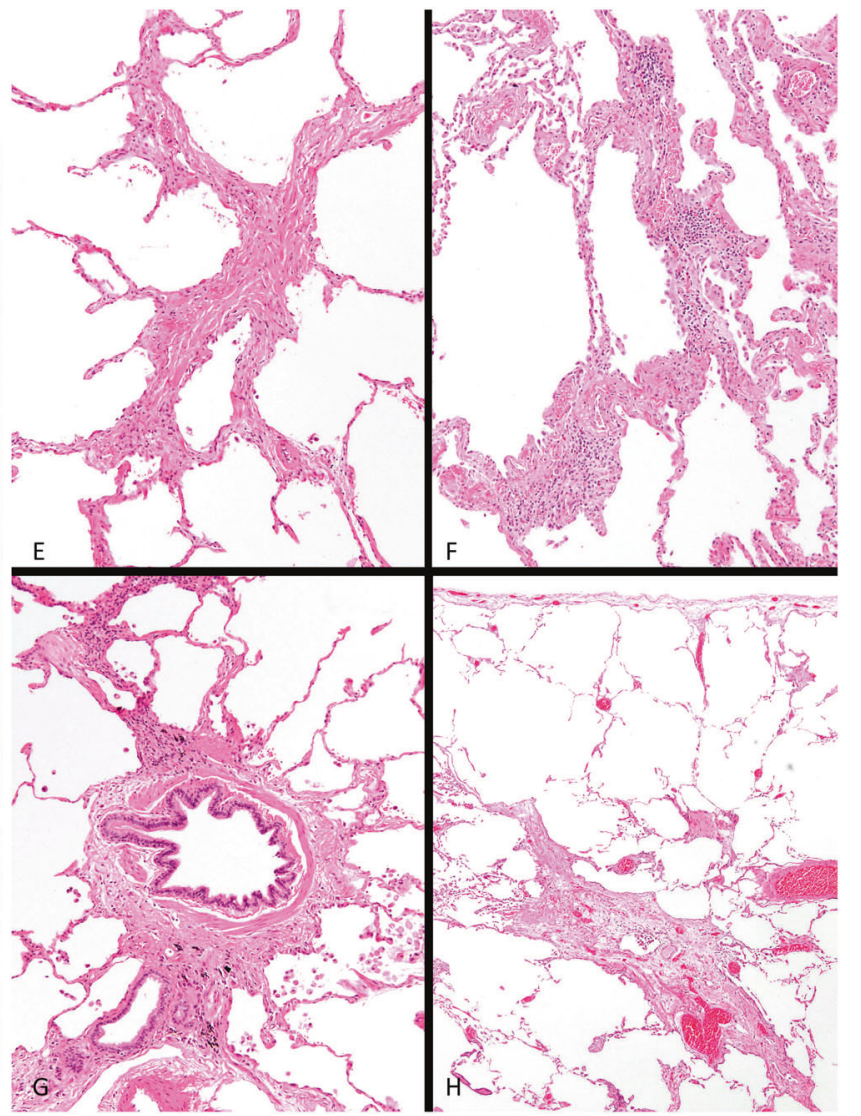

Idiopathic pulmonary fibrosis cases had a statistically greater amount of subpleural fibrosis compared to hypersensitivity pneumonitis cases. e, f Paucicellular interstitial inflammation (e) and mild interstitial inflammation (f). There were no differences in the intensity of interstitial inflammation between IPF and hypersensitivity pneumonitis cases. g Peribronchiolar fibrosis, here around a membranous bronchiole. There were no differences in the frequency/amount of peribronchiolar fibrosis, measured either as a simple qualitative finding, or as a morphometrically determined quantitative value. $\mathbf{h}$ Normal pleura juxtaposed to a bronchiole with peribronchiolar fibrosis. This finding was more common in hypersensitivity pneumonitis cases but did not quite achieve statistical difference from IPF cases

granulomas are generally accepted as features of hypersensitivity pneumonitis, and in this study were found only in cases where the multidisciplinary discussion-established likelihood of hypersensitivity pneumonitis was $50 \%$ or higher, indicating their importance in the final diagnosis. Although we would have accepted airspace giant cells/ granulomas as favoring hypersensitivity pneumonitis, in this set of cases all of the giant cells/granulomas were interstitial. There is less information regarding the diagnostic utility of peribronchiolar metaplasia, but our data suggest that the finding of $>50 \%$ of bronchioles with peribronchiolar metaplasia strongly favors hypersensitivity pneumonitis, although a lesser amount of peribronchiolar metaplasia does not necessarily exclude hypersensitivity pneumonitis. Alternately, one can measure the number of 
foci of peribronchiolar metaplasia per $\mathrm{cm}^{2}$ of tissue; here, a value of $>2 / \mathrm{cm}^{2}$ strongly supported a diagnosis of hypersensitivity pneumonitis.

Conversely, we found that some features previously proposed as supporting a diagnosis of hypersensitivity pneumonitis were not clearly useful. For example, the presence of peribronchiolar fibrosis adjacent to normal pleura was proposed as a marker of hypersensitivity pneumonitis in a previous study [8] and was more frequent here in hypersensitivity pneumonitis cases, but did not quite achieve significance $(p=0.06)$. We also hypothesized that increased peribronchiolar fibrosis around either membranous or respiratory bronchioles would be an indicator of hypersensitivity pneumonitis and that the variability of peribronchiolar fibrosis might be important; however, neither of these were a significant predictor of hypersensitivity pneumonitis. Reassuringly, these results are generally consistent with a recent publication showing that about $1 / 3$ of idiopathic pulmonary fibrosis patients had peribronchiolar fibrosis, although this phenomenon was considerably more frequent in nonidiopathic pulmonary fibrosis patients [19]. We were also unable to show that bridging fibrosis separated idiopathic pulmonary fibrosis and hypersensitivity pneumonitis.

Pathological features favoring idiopathic pulmonary fibrosis included an increased number of fibroblast foci $/ \mathrm{cm}^{2}$ and increased thickness of subpleural fibrosis. Despite this statistical significance, there was considerable overlap between idiopathic pulmonary fibrosis and hypersensitivity pneumonitis and these findings would not be particularly helpful as diagnostic criteria in isolation. A low threshold of $<2$ fibroblast foci $/ \mathrm{cm}^{2}$ may support a diagnosis of hypersensitivity pneumonitis; however, additional data are needed to confirm the reliability of this finding. A very thin pleura would similarly favor hypersensitivity pneumonitis, but measuring pleural thickness is time consuming and generating a set of reference values is probably not practical.

There are few studies in the literature examining the pathologic differences between idiopathic pulmonary fibrosis and hypersensitivity pneumonitis. The most detailed is that of Takemura et al. [9]. They examined 22 cases of fibrotic hypersensitivity pneumonitis, mostly associated with bird exposures, and 13 cases of idiopathic pulmonary fibrosis, and found that centrilobular fibrosis, bridging fibrosis, giant cells/granulomas were statistically more frequent in hypersensitivity pneumonitis. As well they found a number of features that generally would be regarded as part of subacute (nonfibrotic) hypersensitivity pneumonitis including bronchiolitis, "lymphocytic alveolitis" and organizing pneumonia, but we did not observe any of these features of nonfibrotic hypersensitivity pneumonitis in our cases. More important, however, is that, apart from giant cells/granulomas, all the statistically significant features of fibrotic hypersensitivity pneumonitis found by Takemura et al. were also present in some fraction of their idiopathic pulmonary fibrosis cases; the difference between hypersensitivity pneumonitis and idiopathic pulmonary fibrosis cases was the frequency of each feature. This observation emphasizes the major problem of overlapping pathologic findings in these two disease entities, a problem that we have encountered here as well.

This study is limited by our small number of cases, in part because many patients with idiopathic pulmonary fibrosis and hypersensitivity pneumonitis are diagnosed without needing a surgical lung biopsy; nonetheless, many of our comparisons were statistically significant, indicating that we had adequate power for the main comparisons of interest. Clearly the differences that we did find with this small number of cases are strongly predictive, but it is also possible that with larger numbers we would find more statistical differences. By definition, our methodology involves incorporation bias; however, our primary objective was to identify the pathologic variables that form our clinical gestalt, with our goal of generalizing our experience to a broader setting of more diverse physicians. Our referral pattern also results in assessment of complex patients, with surgical lung biopsy reserved for the most challenging patients within this group. For this reason, our patients are likely more difficult to diagnose than average, resulting in more frequent diagnostic uncertainty compared to what would be observed in many cohorts. Finally, our findings were not externally validated, given the challenges in conducting a blinded assessment of lung biopsies from a substantial number of patients.

Lastly, it is important to note that even after a combined clinical-radiologic-pathologic assessment, only 15 of our 23 patients were thought to have a diagnostic likelihood of $70 \%$ or greater (5 usual interstitial pneumonia, 10 hypersensitivity pneumonitis). In other words, $35 \%$ of our patients had a provisional low-confidence diagnosis that was essentially indeterminate [16]. This lack of confidence suggests a frequent overlap in morphology findings for these diagnoses and illustrates the need not just for diagnostic criteria for hypersensitivity pneumonitis, but also indicates the need for more specific diagnostic tests for each of these diseases that can be incorporated into future diagnostic criteria.

\section{Compliance with ethical standards}

Conflict of interest CJR has received grant support and honoraria from Boehringer Ingelheim and Hoffmann-La Roche. AC has received lecture honoraria from Boehringer Ingelheim and Hoffmann-La Roche.

Publisher's note Springer Nature remains neutral with regard to jurisdictional claims in published maps and institutional affiliations. 


\section{References}

1. Travis WD, Costabel U, Hansell DM, King TE Jr., Lynch DA, Nicholson AG, et al. An official American Thoracic Society/ European Respiratory Society statement: update of the international multidisciplinary classification of the idiopathic interstitial pneumonias. Am J Respir Crit Care Med. 2013;188:733-48.

2. Guler SA, Ellison K, Algamdi M, Collard HR, Ryerson CJ. Heterogeneity in unclassifiable interstitial lung disease. A systematic review and meta-analysis. Ann Am Thorac Soc. 2018;15:854-63.

3. Raghu G, Remy-Jardin M, Myers JL, Richeldi L, Ryerson CJ, Lederer DJ, et al. Diagnosis of idiopathic pulmonary fibrosis. An official ATS/ERS/JRS/ALAT clinical practice guideline. Am J Respir Crit Care Med. 2018;198:e44-68.

4. Morell F, Villar A, Montero MA, Munoz X, Colby TV, Pipvath S, et al. Chronic hypersensitivity pneumonitis in patients diagnosed with idiopathic pulmonary fibrosis: a prospective case-cohort study. Lancet Respir Med. 2013;1:685-94.

5. Walsh SL, Calandriello L, Sverzellati N, Wells AU, Hansell DM, Consort UIPO. Interobserver agreement for the ATS/ERS/JRS/ ALAT criteria for a UIP pattern on CT. Thorax. 2016;71:45-51.

6. Walsh SL, Wells AU, Desai SR, Poletti V, Piciucchi S, Dubini A, et al. Multicentre evaluation of multidisciplinary team meeting agreement on diagnosis in diffuse parenchymal lung disease: a case-cohort study. Lancet Respir Med. 2016;4:557-65.

7. Lynch DA, Sverzellati N, Travis WD, Brown KK, Colby TV, Galvin JR, et al. Diagnostic criteria for idiopathic pulmonary fibrosis: a Fleischner Society White Paper. Lancet Respir Med. 2018;6:138-53.

8. Churg A, Bilawich A, Wright JL. Pathology of chronic hypersensitivity pneumonitis what is it? What are the diagnostic criteria? Why do we care? Arch Pathol Lab Med. 2018;142: 109-19.

9. Takemura T, Akashi T, Kamiya H, Ikushima S, Ando T, Oritsu $\mathrm{M}$, et al. Pathological differentiation of chronic hypersensitivity pneumonitis from idiopathic pulmonary fibrosis/usual interstitial pneumonia. Histopathol. 2012;61:1026-35.

10. Hashisako M, Tanaka T, Terasaki Y, Uekusa T, Achcar RD, Aswad BI, et al. Interobserver agreement of usual interstitial pneumonia diagnosis correlated with patient outcome. Arch Pathol Lab Med. 2016;140:1375-82.

11. Churg A, Wright JL, Ryerson CJ. Pathologic separation of chronic hypersensitivity pneumonitis from fibrotic connective tissue disease-associated interstitial lung disease. Am J Surg Pathol. 2017;41:1403-19.

12. Morisset J, Johannson KA, Jones KD, Wolters PJ, Collard HR, Walsh SLF, et al. Identification of diagnostic criteria for chronic hypersensitivity pneumonitis: an International Modified Delphi Survey. Am J Respir Crit Care Med. 2018;197:1036-44.

13. Fischer A, Antoniou KM, Brown KK, Cadranel J, Corte TJ, du Bois RM, et al. An official European Respiratory Society/American Thoracic Society research statement: interstitial pneumonia with autoimmune features. Eur Respir J. 2015;46:976-87.

14. Bolender RP, Hyde DM, Dehoff RT. Lung morphometry: a new generation of tools and experiments for organ, tissue, cell, and molecular biology. Am J Physiol. 1993;265:L521-48.

15. Walsh SLF, Maher TM, Kolb M, Poletti V, Nusser R, Richeldi L, et al. Diagnostic accuracy of a clinical diagnosis of idiopathic pulmonary fibrosis: an international case-cohort study. Eur Respir J. 2017;50:1700936.

16. Ryerson CJ, Corte TJ, Lee JS, Richeldi L, Walsh SLF, Myers JL, et al. A standardized diagnostic ontology for fibrotic interstitial lung disease. An international working group perspective. Am J Respir Crit Care Med. 2017;196:1249-54.

17. Silva CI, Muller NL, Lynch DA, Curran-Everett D, Brown KK, Lee $\mathrm{KS}$, et al. Chronic hypersensitivity pneumonitis: differentiation from idiopathic pulmonary fibrosis and nonspecific interstitial pneumonia by using thin-section CT. Radiology. 2008;246:288-97.

18. Churg A, Sin DD, Everett D, Brown K, Cool C. Pathologic patterns and survival in chronic hypersensitivity pneumonitis. Am J Surg Pathol. 2009;33:1765-70.

19. Tanizawa K, Ley B, Vittinghoff E, Elicker BM, Henry TS, Wolters PJ, et al. Significance of bronchiolocentric fibrosis in patients with histopathological usual interstitial pneumonia. Histopathol. 2019;74:1088-97.

20. Flaherty KR, King TE Jr., Raghu G, Lynch JP 3rd, Colby TV, et al. Idiopathic interstitial pneumonia: what is the effect of a multidisciplinary approach to diagnosis? Am J Respir Crit Care Med. 2004;170:904-10. 